\title{
Low-dimensional Multimodal Deformable Registration of MRI Brain Images in Stereotaxic Space
}

\author{
Daniel Schwarz ${ }^{1}$, Ivo Provazník ${ }^{2}$ \\ 1. Institute of Biostatistics and Analyses, Masaryk University, Czech Republic, \\ 2. Department of Biomedical Engineering, Brno University of Technology, Czech Republic
}

\begin{abstract}
Deformable image registration is a fundamental technique in computational neuroanatomy. An iterative multilevel block matching technique with the use of several recent inventions is proposed here. A symmetric multimodal similarity measure allows to register subject images to an arbitrary digital brain atlas. Smooth deformations produced by scattered data interpolation based on compactly supported radial basis functions suppress gross inter-subject differences and preserve the localized anatomical variability which may be further studied with selected automated morphometry methods. Four similarity measures are tested in an experiment with image data obtained from the Simulated Brain Database and a quantitative evaluation of the algorithm is presented.
\end{abstract}

Keywords: image processing, image registration, MRI images, computational neuroanatomy, radial basis functions

\section{Introduction}

One of widely applied methods in computational neuroanatomy is a voxel-based morphometry (VBM), which has recently become a subject of discussion [1], [2]. It interrogates anatomical MRI scans on voxel by voxel basis, in order to demarcate regions with significant anatomical differences between a group of patients and a control group. Several image preprocessing steps are included in the method's pipeline and deformable image registration is the one playing a crucial role. Its central idea is to find local forces which will deform a floating image to make it more similar to a reference image. The involved non-linear transforms are either based on smooth basis functions [3], [4], [5] or they are physically interpreted, e.g. by mechanics of continuum [6], [7], [8]. The former group of methods produces smooth low-dimensional deformations which are able to suppress only gross anatomical inter-subject differences, whereas the goal of the latter methods is to achieve a perfect match. In [9], [10], images in VBM are put into a stereotaxic space by an affine transform and then they are warped to the reference image by low-dimensional parametric deformations based on lowest-frequency components of discrete cosine transform. The coefficients are searched in an optimization algorithm which minimizes the residual squared difference between the images and simultaneously maximizes the smoothness of the deformations. Only one scaling parameter is incorporated to count for differences in intensities of the images, what makes it suitable for monomodal images only. 
In this paper, we propose a deformable registration algorithm proper for multimodal images. Below, we first explain the methods used in our algorithm and then we present experimental results obtained from its evaluation.

\section{Methods}

Our deformable registration is performed by a multilevel block-matching technique, see fig 1 . A floating image $N$ is deformed to match a reference image $M$ in an iterative process. A resulting displacement $\mathbf{u}$ is made up from local translations of the blocks of the floating image $N$ by radial basis function (RBF) interpolation. The translations representing warping forces $\mathbf{f}$ are found by maximizing symmetric regional similarity measures. The floating image $N$ is assumed to be brought into the same coordinate space as the reference image $M$ by a previous linear registration step.

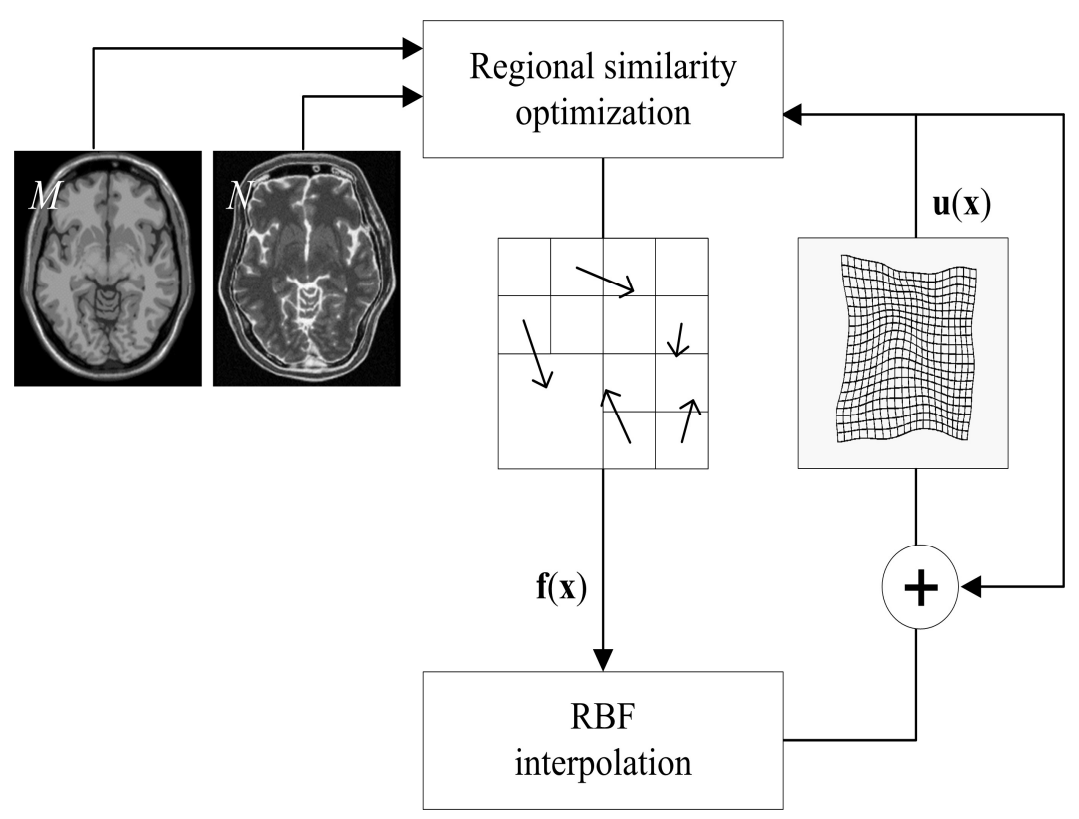

Fig. 1. Deformable registration scheme (see the text for details).

\section{Regional symmetric similarity measures}

Various multimodal similarity measures are examined here. Regional similarity is computed by simply averaging point similarities over the region [11]:

$S_{W} \mathbf{w} \square \frac{1}{K_{W}} \sum_{\mathbf{x} \backslash W} S \mathbf{x}$,

where $S_{W}$ denotes a similarity measure of a region $W$ with the center point $\mathbf{w}$ and $K_{W}$ overlapping voxels $\mathbf{x}$ in which point similarities $S$ are computed. The point similarity measure $S_{M I}$ derived from the well known global similarity measure mutual information is defined by [11]: 


$$
S_{M T}(\mathbf{x})=\log _{2} \frac{p_{M N}(m(\mathbf{x}), n(\mathbf{x}))}{p_{M}(m(\mathbf{x})) \cdot p_{N}(n(\mathbf{x}))},
$$

where $p_{M N}$ denotes joint distribution of intensities and $p_{M}, p_{N}$ are marginal intensity distributions of the images $M$ and $N$ respectively. Another point similarity measure $S_{U H}$ is proposed in [6]:

$$
S_{U H}(\mathbf{x})=S_{H}(\mathbf{x})+S_{M I}(\mathbf{x})=\log _{2} p_{M N}(m(\mathbf{x}), n(\mathbf{x}))+S_{M I}(\mathbf{x})
$$

where $S_{H}$ is a point similarity measure derived from the global joint entropy of the images. All the defined point similarity measures depend on the joint intensity distribution, which is estimated from the joint histogram, which is not known until the images are perfectly matched. Thus, it is usually estimated from the images aligned by the previous registration step. In this way, the deformable registration is done also in [12], where a region similarity measure based on conditional probabilities is proposed. It is rewritten here as another point similarity measure:

$$
S_{P C}(\mathbf{x})=p(n(\mathbf{x}) \mid m(\mathbf{x}))
$$

which is defined by the probability of a correspondence between a given intensity $m$ of the reference image $M$ and any intensity $n$ of the floating image $N$. The conditional probability densities are extracted by normalizing the values of each row of the joint histogram parallel to the axis with the intensities of the floating image $N$. Another similarity measure depending on probability rather than uncertainty is derived here from (2):

$$
S_{P M I}(\mathbf{x})=\frac{p_{M M V}(m(\mathbf{x}), n(\mathbf{x}))}{p_{M}(m(\mathbf{x})) \cdot p_{N}(n(\mathbf{x}))} .
$$

At each level of subdivision, translations of rectangular blocks of the floating image $N$ are searched in an optimization algorithm, which maximizes a selected region similarity measure. Inspired by symmetric registration proposed in [13], the symmetric regional similarity measure is obtained here as a sum of two partial similarity measures. These are computed in the blocks of the floating image according to the reference image blocks as well as in the reverse direction.

To avoid getting trapped in local minima, a combination of extensive search and hillclimbing algorithms is used here. First, a space of all possible translations is searched with a relatively large step. $P$ best points are then used as start points for the following hillclimbing. The minimum of $P$ local minima obtained by the hillclimbing is then declared as the global minimum. 


\section{Multilevel deformation}

Once the local translations are found, the displacement $\mathbf{u}$ is computed separately for each of $D$ dimensions by interpolation with the use of RBF by:

$$
u_{k}(\mathbf{x})=\sum_{i=1}^{B}\left(\alpha_{i} \cdot R\left(\left\|\mathrm{x}-\mathrm{w}_{i}\right\|\right), \quad k=1 \ldots D\right.
$$

where $u_{k}(\mathbf{x})$ is the displacement of a grid point $\mathbf{x}$ in the $k^{\text {th }}$ dimension, $R$ is the radial basis function of the distance $\left\|\mathbf{x}-\mathbf{w}_{\mathrm{i}}\right\|$ between the grid point $\mathbf{x}$ and the center of the $i^{\text {th }}$ block $\mathbf{w}_{\mathrm{i}}$. The coefficients $\alpha_{\mathrm{i}}$ are computed by putting the translations $\mathbf{f}$ into (6) and solving the resulting linear system of $B$ equations separately for each dimension $k$. The compactly supported Wendland's RBF, which was successfully used for landmark-based deformable registration in [4] is used here. Its mathematical properties hold for different spatial support, which is important for the multilevel strategy. For each level of subdivison, the block size is set to the half of the size at the previous level. The displacements are gradually incremented over all levels, refining the resulting deformation in the coarse-to-fine manner. The regions containing poor contour or surface information can be eliminated from the matching process and the algorithm can be accelerated in this way. The subdivision is performed only if at least one voxel in the current region has its normalized gradient image intensity bigger then a certain threshold, see fig. 2 .

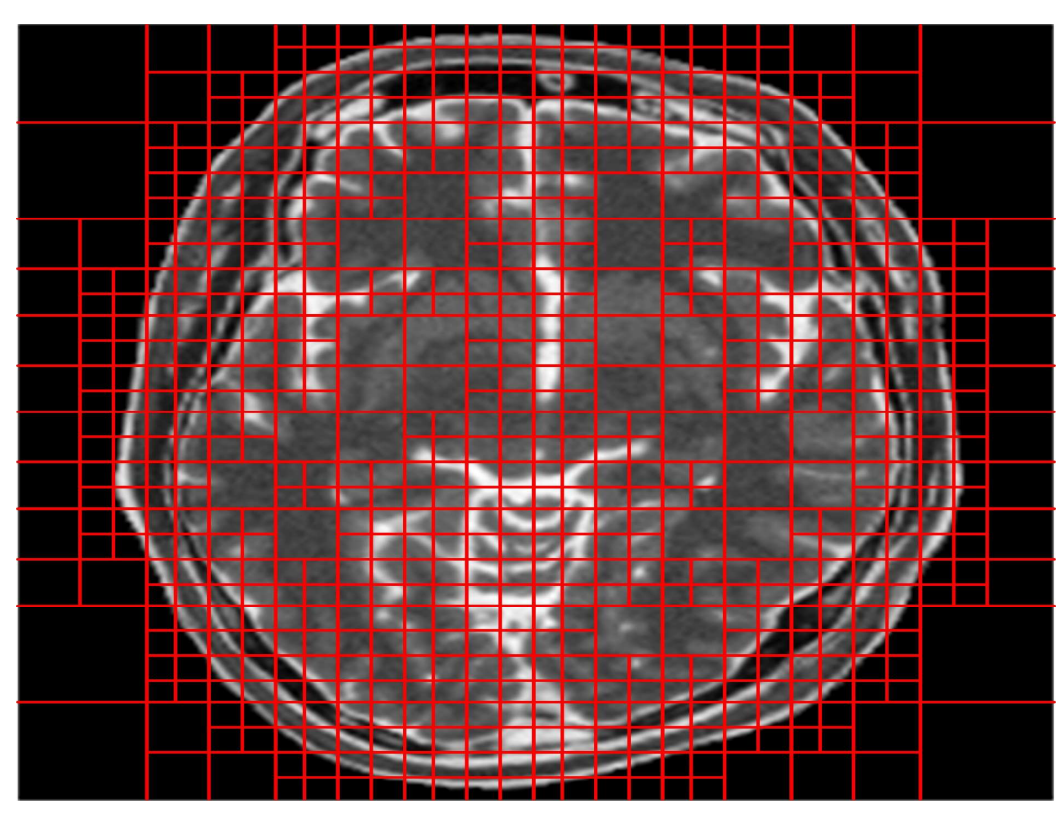

Fig. 2. Illustration of five-level adaptive subdivision.

\section{Tissue probability maps}

The computation of the global joint histogram is not the only way how to estimate the joint intensity distribution of the images $M$ and $N$. When the registration is done in a stereotaxic 
space, tissue probability maps are often available, representing a kind of prior information, which can be used. Here, another estimate of the joint intensity distribution is made with their use. It is further combined with the usual estimate obtained from the global joint histogram by a weighting parameter $\lambda$ :

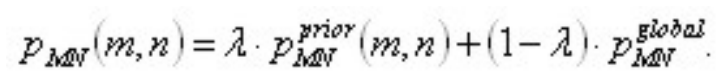

The intensities representing main tissues in the brain images are emphasized in this way.

The joint intensity estimates are re-computed in the each iteration of the registration algorithm. As the intensities of the floating image are not spread on a regular grid, simple unitary increments of individual histogram bins cannot be done. Instead of that, all histogram bins corresponding to the reference image voxels in the neighborhood of a displaced floating image voxel are increased by a value equal to the value of an interpolation kernel function. Cubic B-splines are used for that purpose in the generalized partial volume interpolation (GPVE) described in [14]. This approach is used here to compute the global joint histogram. In the computation of the joint distribution estimate based on the tissue probability maps, the interpolation has to be done among the voxels of the floating image. Thus, some ideas from the distance-weighted scattered data interpolation methods were adapted here and the locally bounded kernel function described in [15] is used in the partial volume interpolation scheme.

\section{Experiment and results}

The performance of the proposed algorithm with various similarity measures was evaluated with the use of 2D image data obtained from Simulated Brain Database (SBD) [16]. The original size of the SBD transversal slices is $181 \times 217$ pixels with the pixel size $1 \times 1 \mathrm{~mm}$. For the evaluation purposes, the images were padded to the size of $217 \times 217$ pixels. The square image size is convenient for the subdivision scheme. Synthetic deformations were composed from random translations at $10 \%$ randomly selected pixels in transversal slices. These force fields were smoothed by gaussian filtering with random standard deviation $(\sigma=10 \pm 5 \mathrm{~mm})$ to obtain final displacements which were then applied on 20 intensity images and corresponding segmented images. The average initial overlap error was $41.0 \%$. The deformations were then recovered by the proposed deformable registration algorithm and the average decrease of the overlap error $\Delta e$ was computed. T1 weighted, T2-weighted and PD (proton density) images with $3 \%$ noise and $20 \%$ intensity nonuniformity were used as floating images and the T1weighted image with no noise and no intensity nonuniformity was used as the reference image. The results for various levels of decomposition are summarized in tab 1. The overlap error got smaller up to the $5^{\text {th }}$ level, when the subimages had size of $7 \times 7$ pixels. Although the next level gave an increase in the global mutual information, the alignment measured by overlap errors and also by visual inspection was constant or worse. This level was considered as the maximum subdivision level for this algorithm. 
Tab. 1. Quantitative validation of the proposed algorithm. The averages of the overlap error decrease $\Delta$ e were computed for various similarity measures, various image pairs and various levels of decomposition.

\begin{tabular}{|c|c|c|c|c|}
\hline \multirow{2}{*}{ Images } & \multicolumn{4}{|c|}{$\Delta \mathrm{e}[\%]$} \\
\hline & $S_{P C}$ & $\boldsymbol{S}_{U H}$ & $S_{M I}$ & $\boldsymbol{S}_{P M I}$ \\
\hline \multicolumn{5}{|l|}{$1^{\text {st }}$ level } \\
\hline $\mathrm{T} 1-\mathrm{T} 1$ & 5.2 & 1.6 & 1.7 & 1.5 \\
\hline T1-T2 & 5.3 & 1.3 & 1.4 & 2.8 \\
\hline T1-PD & 4.4 & 1.5 & 1.3 & 2.2 \\
\hline \multicolumn{5}{|l|}{$2^{\text {nd }}$ level } \\
\hline $\mathrm{T} 1-\mathrm{T} 1$ & 12.6 & 8.3 & 8.4 & 8.5 \\
\hline $\mathrm{T} 1-\mathrm{T} 2$ & 12.3 & 4.7 & 4.8 & 7.4 \\
\hline T1-PD & 10.1 & 5.6 & 6.0 & 5.8 \\
\hline \multicolumn{5}{|l|}{$3^{\text {rd }}$ level } \\
\hline $\mathrm{T} 1-\mathrm{T} 1$ & 22.6 & 17.9 & 17.9 & 19.5 \\
\hline T1-T2 & 21.2 & 9.8 & 9.8 & 16.4 \\
\hline T1-PD & 17.1 & 11.4 & 12.3 & 12.2 \\
\hline \multicolumn{5}{|l|}{$4^{\text {th }}$ level } \\
\hline $\mathrm{T} 1-\mathrm{T} 1$ & 27.2 & 24.8 & 24.9 & 26.6 \\
\hline T1-T2 & 25.3 & 16.0 & 16.2 & 23.3 \\
\hline T1-PD & 20.2 & 15.6 & 16.4 & 17.4 \\
\hline \multicolumn{5}{|l|}{$5^{\text {th }}$ level } \\
\hline $\mathrm{T} 1-\mathrm{T} 1$ & 27.7 & 26.8 & 27.1 & 28.5 \\
\hline T1-T2 & 25.1 & 19.4 & 19.9 & 24.9 \\
\hline T1-PD & 20.0 & 16.9 & 17.9 & 19.2 \\
\hline
\end{tabular}

\section{Conclusion}

An algorithm for low-dimensional atlas-based registration of MRI images was presented. Four various symmetric region similarity measures were studied in an experiment in which synthetic deformations were recovered and the performance of the algorithm was quantified by the decrease of overlap error in segmented images. The similarities were measured with the use of joint histogram and tissue probability maps from MRI brain atlases. The overlap error was lower with the similarity measures $S_{P C}$ and $S_{P M I}$ depending on probabilities than when the similarity measures $S_{M I}$ and $S_{U H}$ depending on uncertainty were used. In our implementation, partial volume interpolation scheme was used, so that it was unnecessary to compute the deformed floating image during the registration process. The proposed algorithm is suitable for the voxel based morphometry, as the precision of the registration can be controlled by the maximum level of decomposition. Thus, only gross inter-subject anatomical differences can be suppressed and the important variability for statistical parametric tests can 
be preserved. In addition, the use of multimodal similarity measure allows to use an arbitrary available brain atlas without any need to transform intensities in the images to obtain monomodal data.

\section{Acknowledgement}

This work has been partly supported by the grant projects 1ET2085120511 AC CR, 102/04/0472 and 305/04/1385 from GACR, and the Research Programme of Brno University of Technology MSM 0021630513.

\section{References}

[1] Friston, K. J., Ashburner,J.: Generative and Recognition Models for Neuroanatomy. NeuroImage, vol. 23, pp. 21-24, 2004.

[2] Davatzikos, Ch.: Why Voxel-based Morphometric Analysis Should Be Used with Great Caution When Characterizing Group Differences. NeuroImage, vol. 23, pp. 1720, 2004.

[3] Pauchard, Y., Smith M. R., Mintchev M. P.: Modeling Susceptibility Difference Artifacts Produced by Metallic Implants in Magnetic Resonance Imaging with PointBased Thin-Plate Spline Image Registration. In Proc. 26th Conf. IEEE EMBS, 2004, pp. 1766-1769.

[4] Fornefett, M., Rohr, K., Stiehl, H. S.: Radial Basis Functions with Compact Support for Elastic Registration of Medical Images. Image Vision Comput., vol. 19, pp. 87-96, 2001.

[5] Rohlfing, T., Maurer, C. R., Bluemke D. A., Michael J. A.: Volume-Preserving Nonrigid Registration of MR Breast Images Using Free-Form Deformation With an Incompresibility Constraint. IEEE Trans. on Medical Imaging, vol. 12, pp. 730-741, 2003.

[6] Rogelj, P., Kovačič, S., Gee, J. C.: Point Similarity Measures for Non-rigid Registration of Multi-modal Data. Computer Vision and Image Understanding, vol. 92, pp. 112-140, 2003.

[7] Tang, S., Jiang, T.: Fast Nonrigid Medical Image Registration by Fluid Model. In: Proc. 6th Asian Conference on Computer Vision, 2004.

[8] Christensen, G. E., He, J.: Consistent Nonlinear Elastic Image Registration. In: IEEE Proc. MMBIA, 2001, pp. 37-43.

[9] Ashburner, J., Friston, K. J.: Voxel-Based Morphometry - The Methods. NeuroImage, vol. 11, pp. 805-821, 2000.

[10] Ashburner, J., Friston, K. J.: Nonlinear Spatial Normalization Using Basis Functions. Human Brain Mapping, vol. 7, pp. 254-266, 1999.

[11] Rogelj, P., Kovačič, S.: Point Similarity Measure Based on Mutual Information. In Biomedical Image Registration: revised papers, 2003, pp.112-121.

[12] Maintz, J. B. A., Meijering, E. H. W., Viergever M. A.: General Multimodal Elastic Registration based on Mutual Information. In Medical Imaging: Image Processing (Proc. of SPIE, vol. 3338), 1998, pp. 144-154. 
[13] Rogelj, P., Kovačič, S.: Symmetric Image Registration. In Medical Imaging: Image Processing (Proc. of SPIE, vol.5032), 2003, pp. 334-343.

[14] Chen, H., Varshney, P. K.: Mutual Information-Based CT-MR Brain Image Registration Using Generalized Partial Volume Point Histogram Estimation. IEEE Trans. on Medical Imaging, vol. 22, pp. 1111-1119, 2003.

[15] Franke, R. Nielson, G.: Smooth Interpolation of Large Sets of Scattered Data. International Journal for Numerical Methods in Engineering, vol. 15, pp. 1691-1704, 1980 .

[16] Collins, D.L., Zijdenbos, A.P., Kollokian, V., Sled, J.G., Kabani, N.J., Holmes, C. J., Evans A. C.: Design and Construction of a Realistic Digital Brain Phantom. IEEE Trans. on Medical Imaging, vol. 17, pp. 463-468, 1998. 